Archives

$8 \mid 1991$

Varia

\title{
Le chômage et son histoire aux États-Unis
}

\section{Alexandre Keyssar}

\section{(2) OpenEdition}

\section{Journals}

Édition électronique

URL : http://journals.openedition.org/ccrh/2830

DOI : $10.4000 /$ ccrh. 2830

ISSN : 1760-7906

\section{Éditeur}

Centre de recherches historiques - EHESS

\section{Édition imprimée}

Date de publication : 15 octobre 1991

ISSN : 0990-9141

\section{Référence électronique}

Alexandre Keyssar, "Le chômage et son histoire aux États-Unis », Les Cahiers du Centre de Recherches Historiques [En ligne], 8| 1991, mis en ligne le 18 mars 2009, consulté le 10 décembre 2020. URL http://journals.openedition.org/ccrh/2830 ; DOI : https://doi.org/10.4000/ccrh.2830

Ce document a été généré automatiquement le 10 décembre 2020.

Article L.111-1 du Code de la propriété intellectuelle. 


\title{
Le chômage et son histoire aux États- Unis
}

\author{
Alexandre Keyssar
}

1 Quoiqu'en pensent les économistes américains, dont les théories sont antihistoriques et ignorent l'histoire, le phénomène du chômage a une longue histoire aux États-Unis. Cette dernière se divise en trois périodes. La première (vers 1820-1870) a vu l'émergence du phénomène moderne du chômage. La deuxième (vers 1870-1935) pourrait s'appeler «l'ère de l'incertitude ». La troisième enfin, qui va de 1935 à nos jours, est une période de gestion du chômage; elle se subdivise en deux sous-périodes importantes, la seconde commençant en 1973.

2 I. Le fait même de parler d'une période d'émergence suggère que le chômage n'a pas existé - ou du moins qu'il y en avait très peu - avant 1820. Cette proposition est globalement correcte. Il y eut bien sûr, au cours du xviIIe siècle, des gens qui se trouvaient sans travail, mais le phénomène du chômage, au sens moderne du terme, à savoir le fait que des gens qui veulent ou ont besoin de travailler ne trouvent pas de travail, n'était ni très manifeste, ni chronique ou systématique. Les raisons en sont moins dans le comportement de l'économie que dans la structure sociale et dans la nature des rythmes de travail dans un monde artisanal et agricole ${ }^{1}$.

3 En effet, dans le Sud du pays, les esclaves bien évidemment n'étaient pas exposés au chômage. Dans le Nord, les artisans et les paysans - qui constituaient une grande partie de la population active - contrôlaient leurs propre temps et rythmes de travail ; vivant sur le rythme annuel des saisons, ils pouvaient organiser et alterner les tâches afin d'éviter des périodes de désœuvrement total et involontaire. En conséquence, pendant toute l'époque antérieure à la Guerre Civile et surtout avant la décennie 1830, les allusions au problème du chômage sont extrêmement rares, même dans les écrits des ouvriers.

4 Deux transformations critiques mirent un terme à ce silence. La première fut la révolution industrielle, qui transforma en ouvriers et salariés la majeure partie de la main d'œuvre. Entre 1820 et 1870, le pourcentage des salariés dans la population active 
augmenta fortement - même si cet accroissement reste difficile à évaluer. Un nombre de plus en plus important de travailleurs devint ainsi dépendant des patrons pour leur emploi et leurs salaires et le temps de travail dont ils ne contrôlaient ni la durée ni l'aménagement. En liaison avec ce premier phénomène, une seconde transformation fut la séparation, la coupure d'avec la terre, de ceux qui travaillaient dans l'industrie. Ce processus commença avec la différenciation géographique des secteurs économiques aux États-Unis, et s'acheva avec la vague d'immigration, surtout irlandaise, entre 1840 et 1860. La conséquence en fut la diminution de la capacité des ouvriers travaillant dans l'industrie de retourner à la terre, à la ferme, quand l'emploi industriel venait à manquer. De plus, l'urbanisation ne leur permettait pas de disposer de jardins qui les auraient aidés à survivre pendant les périodes de désœuvrement. De ce fait, les travailleurs, les salariés, furent de plus en plus dépendants d'un emploi quotidien et hebdomadaire, et de plus en plus nécessiteux quand ils n'avaient pas d'emploi dans l'industrie.

5 Ces processus étant bien en place, dans le Nord-Est du moins, la dépression cyclique de 1873 à 1878 put devenir la scène sur laquelle plusieurs événements symboliques importants se sont produits. Ainsi, le premier essai systématique de dénombrement des chômeurs. Ce premier essai eut lieu dans l'État du Massachussets en 1878, puis dans l'ensemble du pays en 1880 . Avec ces tentatives, la définition moderne du mot chômeur fit son apparition, suivie en 1887 de celle du mot chômage. Ces événements statistiques et linguistiques ont été les prémices, un peu tardives certes, de l'émergence du phénomène moderne du chômage. De ce fait, ils ont inauguré « l'ère de l'incertitude »².

6 Cette période - qui a duré jusqu'à 1935 et qui a vu le triomphe du capitalisme industriel aux États-Unis - se caractérise, surtout dans la classe ouvrière, par un chômage continuel et chronique, pendant les crises comme dans les années de prospérité. Le taux annuel du chômage s'élève de 4-6\% pendant les meilleures années jusqu'à $12-15 \%$ pendant les dépressions (Tableau $\mathrm{n}^{\circ} 1$ ). Mais un chiffre plus important et plus révélateur que le taux de chômage est celui que j'ai appelé the annual unemployment frequency - le pourcentage de la population active qui, au cours de l'année, s'est trouvée au chômage pendant au moins un mois $^{3}$. Le profil de ce chiffre est bien différent : pendant les années de prospérité, il y avait un minimum de 15 à $20 \%$ de la population active qui faisait l'expérience du chômage ; pendant les périodes de dépression, la frequency montait jusqu'à 30-40\% (Tableau $\mathrm{n}^{\circ}$ 2).

7 En général, la durée moyenne du chômage était de 3 à 4-5 mois ${ }^{4}$. Une grande majorité des chômeurs appartenait à la classe ouvrière et le taux de chômage était beaucoup plus élevé chez les ouvriers que dans les classes moyennes. Mais ce taux de chômage - et en termes psychologiques la menace de chômage - n'était pas du tout identique dans les différents corps de métiers : ainsi, les ouvriers de la chaussure étaient beaucoup plus souvent au chômage que ceux du textile. Plus surprenant, peut-être, est le fait que la répartition du chômage était assez égalitaire dans les différents groupes sociaux de la classe ouvrière. Les immigrés connaissaient davantage le chômage que ceux qui étaient nés dans le pays, mais la différence n'était pas très importante. De la même façon, le taux de chômage des jeunes n'était guère plus élevé que celui des adultes. Et, en général, les hommes et les femmes ont été touchés par ce phénomène de la même façon pendant toute cette période ${ }^{5}$

8 Le problème du chômage était en fait si répandu qu'il suscita toute une série de tentatives pour le guérir ou le résoudre, tentatives très limitées du fait de l'absence de solution évidente dans le cadre d'une économie capitaliste. L'option de créer plus de travail - qui 
est devenue plus tard l'option keynésienne - était difficile à envisager au XIX ${ }^{\mathrm{e}}$ siècle. L'idée de payer des gens sans travail restait en dehors des normes sociales. Et la redistribution ou partition du travail n'était finalement - comme l'ont fait remarquer certains critiques contemporains - qu'une façon de partager la misère. Il y eut néanmoins des tentatives, des mouvements politiques et sociaux, de trois types différents ${ }^{6}$. Les mouvements de chômeurs en furent un premier aspect. D'une façon générale, ils réclamaient l'aide de l'État (ville, État ou gouvernement fédéral). Ces mouvements furent souvent flamboyants et déstabilisants, et toujours de courte durée ; la malheureuse armée de Jacob Coxey en 1894 en est l'exemple le plus connu. La deuxième force organisée contre le chômage fut le mouvement syndical. A la fin du XIX siècle, les syndicats ont désigné le chômage comme le problème le plus important de la classe ouvrière et ils ont fait des efforts vigoureux pour le résoudre. Ils ont proposé la solution relativement égalitaire d'une réduction de la journée de travail à huit heures : les dirigeants syndicaux croyaient qu'une telle solution éliminerait effectivement le chômage. Bien entendu, cette stratégie - qui partait de prémices erronées - a échoué et son échec - manifeste dans les années 1900-1010 - a conduit le mouvement syndical à des stratégies de "sauve qui peut ». Parmi ces méthodes qui ont dominé le mouvement syndical de 1900 à 1930, citons la restriction de l'apprentissage, la tentative de contrôle du marché du travail pour garantir l'emploi aux seuls membres des syndicats; les rudes conflits entre syndicats pour obtenir la « juridiction » sur des métiers différents ; enfin, l'adoption de restrictions légales de l'immigration.

9 Les réformateurs bourgeois ont constitué le troisième centre de réaction politique contre le chômage. Surtout après la crise économique de 1907, ils ont considéré le chômage - surtout les "progressistes »- comme la source d'autres problèmes sociaux. Pour réduire l'impact du problème, ils ont proposé des changements dans les pratiques des entreprises (surtout dans la gestion du personnel) et un système d'assurance pour les chômeurs. Avant 1930, et malgré les activités de ces trois groupes, peu de mesures avaient été prises : il n'y avait pas de système d'assurance ni de politique publique de lutte contre le chômage, et fort peu d'entreprises avaient changé leurs pratiques dans le but de réduire les licenciements. Il y avait plusieurs raisons à cette carence.

1 - l'impulsion au changement était liée aux périodes de dépression, en général de trop courte durée pour donner une assise aux mouvements des réformistes ;

2 - les coalitions politiques en faveur des réformes étaient trop faibles;

3 - les patrons étaient capables de s'entendre sur des initiatives réformistes en créant des marchés secondaires du travail sans réduire le taux de chômage ;

4 - les syndicats, essentiellement l'American Federation of Labor, dirigée jusqu'en 1924 par Samuel Gompers, étaient hostiles à l'assurance-chômage.

10 Tous ces obstacles à une véritable réforme furent balayés par la grande dépression des années 30 , pendant laquelle le taux de chômage fut supérieur à $20 \%$ et l'annual unemployment frequency souvent supérieur à $40 \%$, avec des durées moyennes de chômage d'une année ou plus. Cette crise bien connue a été à l'origine de nombreux et importants changements institutionnels :

$11 \mathbf{1}$ - le gouvernement fédéral s'engagea dans une politique de plein emploi ; cette décision était implicite dans les mesures du New Deal de Franklin Roosevelt et elle fut formalisée avec l'Employment Act de 1946 et la création du Conseil des conseillers économiques ;

122 - un programme d'assurance pour les chômeurs fut mis en place en 1935 ; 
3-l'ancienneté fut retenue - surtout dans les contrats entre les syndicats et les entreprises - comme le principe qui présiderait au choix des licenciements.

Dans leur ensemble, ces changements, qui ont protégé les chômeurs et rationalisé la répartition du chômage, ont effectivement mis un terme à « l'ère d'incertitude ».

II. Pendant la première moitié de la période de gestion du chômage, (vers 1945-1973, ces changements institutionnels ont semblé être un succès : le capitalisme fut préservé (ce qui n'était pas du tout évident en 1934 ou 1937) et l'un des problèmes fondamentaux était circonscrit. Le taux de chômage restait en général d'environ 4-5\%, et même dans les périodes de dépression, il ne fut pas très élevé : $7 \%$ (Tableau $n^{\circ} 1$ ). Dans le même temps, les unemployment frequencies ont baissé, ce qui signifie que la proportion de la population active qui connaissait le chômage chaque année a diminué. De plus, une grande majorité des chômeurs (70 \% à 80 \%) était protégée par l'assurance et l'instauration du principe de l'ancienneté a apporté une sécurité de l'emploi très importante (taux de chômage de $1 \%$ à $2 \%$ ) pour les hommes blancs et adultes. Il y a néanmoins des ombres au tableau. Ainsi, si le principe de l'ancienneté permettait d'assurer la sécurité de l'emploi pour les adultes, il avait comme contrepartie de la diminuer pour les jeunes : après la Deuxième Guerre mondiale, le taux de chômage des jeunes a augmenté. Il en fut de même pour le taux de chômage des noirs et la différence des taux entre les blancs et les noirs est devenue plus importante que celle qui avait pu exister pendant les années 1880-1920 entre les immigrés et ceux qui étaient nés aux États-Unis. De plus, les durées de chômage s'allongèrent : si le pourcentage de la population touchée par le chômage a baissé, en revanche, la durée du chômage a augmenté. La répartition du poids du chômage devenait donc moins égale ${ }^{7}$.

Toutes ces tendances étaient manifestes dans les années 60 mais elles se sont sensiblement accentuées après 1970; une augmentation séculaire du taux de chômage s'est alors produite en même temps que l'aggravation des crises cycliques. Les années 80 ont connu le taux de chômage (et les fréquences) le plus élevé depuis 1939 ainsi qu'un creusement des écarts entre les jeunes et les adultes autant qu'entre les noirs et les blancs. En même temps, à cause des changements législatifs et démographiques, il y eut une diminution graduelle mais ininterrompue de la proportion des chômeurs touchant des allocations pendant leur chômage ${ }^{8}$.

Il est difficile de savoir si tout cela signifie que l'ère de gestion du chômage s'achève sur un échec. Mais il est évident que les politiques des gouvernements fédéraux (y compris la politique keynésienne) n'ont produit aucune amélioration à long-terme des taux de chômage. Il est évident aussi que les années 1980 et 1990 ne ressemblent pas aux années 50 , que ce soit pour la conjoncture ou les mentalités. La conjoncture est plus difficile et plus grave ; on ne croit plus que la société soit capable de résoudre le problème.

18 III. Venons-en maintenant à un aspect remarquable de cette histoire récente : comment se fait-il que la situation ait pu s'aggraver autant sans susciter plus de protestation - dans la classe ouvrière ou chez les réformateurs - contrairement à ce qui s'était produit auparavant? Pourquoi la société américaine a-t-elle toléré en silence cette détérioration ? La réponse comporte deux éléments, me semble-t-il ${ }^{9}$.

Premièrement, la classe ouvrière connaît des divisions, en particulier raciales et ethniques, qui sont accentuées par la répartition du chômage en fonction du principe d'ancienneté. En bref, l'adoption de ce principe a créé une situation où les ouvriers les moins menacés par le chômage (adultes, hommes, blancs) sont ceux qui ont le plus de pouvoir dans les syndicats; de la même façon, les ouvriers qui se sont organisés sont 
moins menacés que ceux (la grande majorité) qui restent en dehors des syndicats. De ce fait, le mouvement syndical ne s'est pas beaucoup intéressé au problème du chômage ou de la sécurité de l'emploi. De plus, la faiblesse économique et politique des syndicats - à l'époque de Reagan et face à la compétition internationale - a renforcé les hésitations à agir.

Dans la bourgeoisie, la tolérance à l'égard du chômage est le résultat de tendances intellectuelles et idéologiques qui ont découragé l'action. Après la Deuxième Guerre mondiale, on pouvait supposer que le gouvernement était responsable, qu'il s'efforçait de diminuer le taux de chômage ; de plus, il y avait des allocations pour les chômeurs; les victimes de licenciement ne risquaient donc pas de devenir des nécessiteux. Jusqu'à très récemment, seuls les experts savaient que le nombre de chômeurs touchant des allocations était en diminution. Dans ces circonstances, il y avait peu de raisons de protester. Il existait d'ailleurs dans certains milieux de la bourgeoisie une autre motivation pour ne pas réagir : l'idée - durable, résistante à toute enquête empirique que le chômage n'était pas vraiment involontaire, que les chômeurs étaient des paresseux et qu'il y avait du travail pour ceux qui voulaient vraiment travailler.

Cette conviction qu'il n'y avait rien à faire - et qu'il ne fallait rien faire - a été renforcée par un ensemble de rationalisations élaborées par les sciences économiques. Depuis 1945, les économistes ont acquis un certain pouvoir et une certaine influence dans la politique américaine, et ils sont responsables des questions du chômage (ce qui n'était pas le cas avant 1940). Les économistes des années 1970 et 1980 ont présenté une série d'arguments et d'analyses qui ont abouti à l'immobilisme.

Tout d'abord, ils ont avancé un argument, résurgence d'un argument traditionnel: le chômage n'existerait pas si les salaires étaient flexibles à la baisse. La version contemporaine de cet argument est que le chômage est généré par les institutions qui restent attachées à la rigidité des salaires - les syndicats par exemple - ou par les lois qui garantissent des salaires minimums. Comme ces institutions et ces lois sont toutes souhaitées et soutenues par la classe ouvrière, l'implication de ces analyses est que le chômage est la faute de la classe ouvrière et donc des chômeurs eux-mêmes ${ }^{10}$.

Une deuxième proposition développée au cours de cette période est qu'une réduction du chômage n'est pas toujours bonne pour la société et l'économie. Cette proposition découle de la courbe de Philips - qui propose un rapport inversement proportionnel entre l'augmentation du taux d'inflation et la diminution du taux de chômage - et de la contribution théorique de Milton Friedman : l'idée d'un "taux naturel de chômage ». Tous deux ont suggéré que le plein emploi conduirait à des niveaux intolérables d'inflation; ce faisant, ils ont suggéré qu'il était possible qu'il n'y ait pas assez de chômage (idée presque impensable en 1900 ou 1930) ${ }^{11}$.

Plus récemment, certains économistes ont conclu que les chiffres du gouvernement ne constituaient pas une bonne mesure du chômage involontaire. En effet, avec l'augmentation graduelle du taux de chômage depuis 1970, un débat s'est instauré entre économistes américains sur l'existence du chômage « involontaire ». Ce débat provient du fait que les chiffres gouvernementaux sur le chômage ne correspondent pas aux modèles mathématiques ou aux objectifs politiques. D'après ces économistes, cela vient nécessairement du fait que les chiffres ne mesurent pas le chômage réel, mais plus précisément qu'ils comptabilisent comme chômeurs des individus qui ne devraient pas être considérés comme de réels chômeurs. Des notions théoriques telle que search theory 
ont donc été élaborées par ces économistes précisément pour dénombrer les véritables chômeurs et expliquer l'allure inattendue des statistiques officielles ${ }^{12}$.

Le fondement empirique de toutes ces notions a toujours été assez faible, mais ces idées - surtout lorsqu'elles se cumulent - permettent de justifier rationnellement l'absence d'une politique de lutte contre le chômage : le chômage n'existe pas ; c'est de leur faute ; c'est volontaire; trop peu de chômage, c'est dangereux. L'acceptation de ces notions favorise l'érosion de la conjoncture, l'attitude de retrait du gouvernement et le silence de ceux qui ne risquent pas de connaître eux-mêmes le chômage. Il est frappant de constater qu'au cours de l'été et de l'automne 1991, le président Bush aurait pu refuser de prolonger les allocations des chômeurs sans rencontrer aucune protestation sérieuse.

En fait, dans la période récente, la gestion du chômage s'est surtout consacrée à la gestion des dimensions politiques du problème. La leçon de la période qui a suivi la guerre est qu'il n'est point nécessaire pour un régime capitaliste d'éliminer ou même de réduire le chômage pour parvenir à la stabilité politique ou la maintenir. Au contraire, l'histoire récente suggère que des niveaux de chômage assez élevés peuvent être tolérés si l'on aide un certain pourcentage de chômeurs, si le nombre des chômeurs n'est pas trop important et si une grande partie d'entre eux appartient à des minorités raciales ou ethniques. Il est difficile de savoir si cette situation - cet équilibre entre les impératifs politiques et les impératifs économiques - peut durer longtemps; la réponse viendra probablement autant de l'Europe et même de l'Europe de l'Est que des États-Unis.

Tableau $n^{\circ} 1$ - Taux de chomâge aux États-Unis (Moyenne sur 5 ans) - 1870-1989

\begin{tabular}{|c|c|c|}
\hline & $\begin{array}{l}\text { Tăux de chômağge } \\
\text { Tarus: moyen }\end{array}$ & 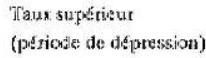 \\
\hline $1876-74^{\circ}$ & 7 & \\
\hline $1875 \% \mathrm{p}^{\mathrm{*}}$ & 12 & $14(18876)$ \\
\hline $18830-344^{\prime \prime}$ & 5 & \\
\hline $18835-89^{\circ}$ & 7 & 8 (1885) \\
\hline $1800-94$ & 8 & $18(18984)$ \\
\hline 189595 & 12 & $15\left(1897^{7}\right)$ \\
\hline $1900-404$ & 4 & $5(1904)$ \\
\hline $1005-105$ & 4 & $8(1908)$ \\
\hline $1910-14$ & 6 & 7 (1911) \\
\hline $1915-19$ & 4 & $9(1915)$ \\
\hline $1920-24$ & 6 & $12(1921)$ \\
\hline $1925-29$ & 3 & \\
\hline $1930-34$ & 19 & $25(1933)$ \\
\hline $1935-39$ & 18 & $19(1938)$ \\
\hline $1940-14.4$ & 6 & \\
\hline $19455-451$ & 4 & $6(1949)$ \\
\hline 1950-54. & 4 & $5(19564)$ \\
\hline 1955599 & 5 & $7(1958)$ \\
\hline $19600-64$ & 6 & $7(1961)$ \\
\hline 1965.459 & A & \\
\hline $1970-74$ & $s$ & $6(1971)$. \\
\hline 1975.75 & 7 & $9(1975)$ \\
\hline $19830-34$ & 8 & 20 (1982) \\
\hline 19835.49 & 6 & \\
\hline
\end{tabular}

". Calculs apperoximatifis de l'auteur.

Sources : Historical Statistics of the United States, p. 135 ; US BLS, Bulletin 2340, p. 129 ; US BLS, Bulletin 2361, p. 3. 
Tableau $n^{\circ} 2$ - Incidence annuelle du chomâge et dur\&e des périodes de chomâge

\begin{tabular}{|c|c|c|c|c|}
\hline Anniée & $\begin{array}{l}\text { Personnes ayant } \\
\text { connu une périod: } \\
\text { de chón:nage clars } \\
\text { l'année (\%) }\end{array}$ & 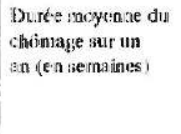 & $\begin{array}{l}\text { Duríe moyesine } \\
\text { p\&r pé riade cle } \\
\text { de chömage } \\
\text { (ent semaints) }\end{array}$ & 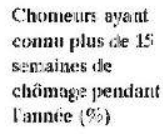 \\
\hline $2835^{x}$ & 30 & 18 & & $57^{7^{\text {it }}}$ \\
\hline $18 \times 0$ & 26 & 15 & & $4.2^{* * * *}$ \\
\hline $18: 5^{x}$ & 28 & 14 & & \\
\hline $29 \times 0$ & $: 2$ & 15 & & $52^{n i k}$ \\
\hline 2901 & 27 & 11 & & \\
\hline 1.9119 & 1.9 & 17 & & \\
\hline .9 .33 & $>35$ & & & \\
\hline $1.931-39$ & & & & $60,-90$ \\
\hline 2.938 & $>\$ 0$ & & & \\
\hline $19: 18$ & & & 17 & 27 \\
\hline $29: 52$ & & & 17 & 2.4 \\
\hline $19: 97$ & 1.5 & & 2.1 & 4.9 (195:) \\
\hline i.9.5:2 & 3.8 & & 29 & 3.6 \\
\hline $19: 7$ & 1.3 & & 17 & 23 \\
\hline 19,59 & & 16 & & \\
\hline .972 & is & & 24 & 344 \\
\hline 1.974 & 1.8 & 20.32 & & \\
\hline .975 & 20 & & & \\
\hline 1.977 & 1.8 & & 29 & $3 i s$ \\
\hline .977 .79 & $14-17$ & 22 & & \\
\hline $29: 37$ & 1.4 & & 29 & 4.5 \\
\hline
\end{tabular}

$\because$ (Massachusetts)

**. Durte de 4 tacis cu plus pour ces années

\section{NOTES}

Sources : A. KEYSSAR, Out of work... pp. 55,146, 302-305; tableaux non publiés du recensement de 1910 ; R.A. MARGO, «The incidence and Donation of Unemployement : Some Long-Term Comparaisons », Economics Letters, 32, 1990, pp. 217-220 ; A.C. BOLINO, « The Duration of Unemployement ; Some Tentative Historical Comparaisons », Quarterly Review of Economics and Business, 6, Summer 1966, pp. 31-47 ; Eleventh U.S. Census, Population, Paris II, pp. 302, 448 ; Twelfth U.S. Census, Occupations, pp. LXXXVII-XIII, p. 214 ; L.H. SUMMERS, Understanding Unemployement, Cambridge, 1990, pp. 21-24; 18 th Annual Report of the U.S. Commission of Labor, pp. 290-97.

1. Voir cette description de l'ère d'émergence dans Alexandre KEYSSAR, Out of Work: The First Century of Unemployment in Massachussets, Cambridge, 1986, pp. 1-38.

2. A. KEYSSAR, Out of Work..., pp. 1-16; cf. Robert SALAIS, Nicolas BAVEREZ et Benedicte REYNAUD, L'Invention du chômage, Paris, 1986, pp. 13-41.

3. Cette période d'un mois a été retenue parce que c'était la durée minimum qui apparaissait dans les recensements à la fin du XIX ${ }^{\mathrm{e}}$ siècle.

4. La frequency peut être convertie en taux si la durée en est connue ; voir A. KEYSSAR, op. cit., pp. 357-8.

5. A. KEYSSAR, op. cit., pp. 39-110, 299-307.

6. Pour une description des mouvements et des résultats de leur action, cf. A. KEYSSAR, op. cit., pp. 177-298. 
7. A. KEYSSAR, «Unemployment Before and After the Great Depression », Social Research, $54, \mathrm{n}^{\circ} 2$, Summer 1987, pp. 203-212.

8. New York Times, December 2, 1990 ; January 28, 1991 ; April 5 et 22, 1991.

9. Cf. A. KEYSSAR, «History and the Problem of Unemployment ", Socialist Review, Fall 1989, pp. 20-28.

10. Voir Martin FELDSTEIN, "The Economics of the New Unemployment ", The Public Interest, 1973, pp. 3-42 ; Richard KRASHEVSKI, «What is So Natural About High Unemployment?», American Economic Review, May 1988, pp. 289-93; Lawrence H. SUMMERS, Understanding Unemployment, Cambridge, 1990, pp.316-22 ; Carl SHAPIRO et Joseph STIGLITZ, «Can Unemployment Be Involontary? Reply », American Economic Review, December 1985, pp. 1215-17.

11. Cf. David H. GORDON, « Six Percent Unemployment Ain't Natural ? », Social Research, Summer 1987, pp. 223-46.

12. Cf. William DARITY, "Beveridge and the New Search Unemployment». Journal of Post Keynesian Economics, Winter 1981-2, pp. 171-80 ; Lorne CARMICHAEL, «Can Unemployment Be Involontary? Comment», American Economic Review, December 1983, pp.1143-52; L. H. SUMMERS, Unemployment, pp. 227-85; Glen CAIN, "The Unemployment Rate as an Economic Indicator ", Monthly Labor review, December 1983, pp. 1143-52; Larry SAMUELSON, «Implicit Contracts with Heterogeneous Labor », Journal of Labor Economics, January 1985, pp. 70-90 ; D. H. GORDON, op cit, pp. 223-46.

\section{AUTEUR}

\section{ALEXANDRE KEYSSAR}

Alexandre KEYSSAR est professeur au Massachussets Institute of Technology ; il a été directeur d'études associé à l'EHESS en 1991. 